\title{
Scattering of second-harmonic light from small spherical particles ordered in a crystalline lattice
}

\author{
Jordi Martorell and R. Vilaseca \\ Department de Física i Enginyeria Nuclear, Universitat Politècnica de Catalunya, 08222 Terrassa, Barcelona, Spain \\ R. Corbalán \\ Department de Física, Universitat Autònoma de Barcelona, 08193 Bellaterra, Barcelona Spain
}

(Received 19 February 1997)

\begin{abstract}
Experimental evidence of scattering of second-harmonic light from the surface of spherical particles of optical dimensions is presented. This mechanism for second-harmonic generation is observed in a suspension of monodisperse spherical colloidal particles, ordered in a centrosymmetric crystalline lattice. In this periodic structure the mechanism of phase matching is provided by the bending of the photon dispersion curve near the Bragg reflection band. A simple theoretical analysis based on the Rayleigh-Gans scattering approximation shows that constructive interference of the second-harmonic light scattered from different portions of a singlesphere surface leads to a nonvanishing field with a quadrupolar distribution intensity pattern. [S1050-2947(97)06506-2]
\end{abstract}

PACS number(s): 42.65.Ky, 42.25.Bs, 42.25.Fx, 78.20.Ci

\section{INTRODUCTION}

Light propagation through inhomogeneous media leads to the distortion of an incoming wave front or the well-known phenomenon of scattering. A general treatment may consider first the single scattering from each disturbance in the medium and then account for the cooperative scattering by the specific spatial arrangement of the disturbances. One of the most relevant consequences of the cooperative scattering from monodisperse spherical particles of optical dimensions ordered in a crystalline lattice is the strong reflection at wavelengths satisfying the Bragg condition [1]. Coherent multiple scattering manifested also clearly in random media with the observation of enhanced backscattering of light incident on a concentrated aqueous suspension of latex microspheres [2]. In the years following this discovery, work was aimed at studying the propagation of light and sound [3] in disordered dielectric materials, such as colloidal particles of $\mathrm{TiO}_{2}$ embedded in polystyrene [4]. Along these lines, several experiments using strongly scattering media were devised in the search for photon localization in the time domain [5]. Coherent scattering may also strongly alter the radiative properties of an oscillating dipole embedded in a crystalline lattice formed by dielectric spheres separated by distances of the order of the wavelength of the emission light [6]. Coherent effects in disordered media were also shown to be responsible for the inhibition and enhancement of spontaneous emission in random media [7] Laser action has also been experimentally demonstrated in such strongly scattering media [8].

Until recently, the study of scattering from particles of optical dimensions was limited for the most part to the linear interaction between the electromagnetic field and the scattering material. Limitations in the study of nonlinear scattering were imposed by the requirement of a high pumping intensity and momentum conservation, as well as for the need of an intrinsic orientation in the material for the observation of a non-negligible lowest-order nonlinear interaction. In 1988, the use of birefringent sheared colloidal crystals for optical second-harmonic generation (SHG) was suggested by Lawandy, Johnston, and Martorell [9] after a macroscopic orientation of nonlinear molecules was observed in such crystals [9]. An alternative mechanism for SHG in colloidal crystals considered more recently [10] and in the present work is based on the constructive interference of the light scattered at the double frequency from different portions of the surface of a spherical particle. At the interface between the sphere surface and the surrounding medium second-order nonlinear processes in the dipole approximation are allowed by the local breaking of the inversion symmetry. As is well known, second-harmonic (SH) light may be reflected from the interface of two centrosymmetric media, due to the lack of inversion symmetry of the surface layers [11], or scattered by orientational fluctuations of molecules that break locally the macroscopic centrosymmetry of a solution [12].

The experimental and theoretical work presented below shows that SHG in the dipole approximation, a process forbidden in the bulk of centrosymmetric crystals is allowed in a crystal composed of spherical particles of optical dimensions because of the local breaking of the inversion symmetry at the surface of each spherical particle. Details of the experimental work and methods used to enhance and measure the second-order nonlinear interaction present at the surface of a dielectric sphere of optical dimensions are given in Sec. II. In the same section, using intuitive arguments based on the theory of Bragg reflection in periodic lattices with a nonlinear material, we show that our experimental measurements of SHG in such nonlinear colloidal crystals indicate phase matching of the fundamental and $\mathrm{SH}$ waves. In Sec. III we present the essential features of a theoretical analysis developed to account for the SH light scattered from dielectric spheres located in the sites of a crystalline lattice. In this model we determine first the spatial distribution of the scattered SH light from a single sphere and then consider the cooperative scattering from all spheres located on a plane normal to the direction of the propagation of the incoming fundamental beam. Finally, predictions from that model are compared with the experimental results of the observed 
second-order nonlinear process. In Sec. IV we discuss the relevance and applications of this nonlinear phenomenon.

\section{EXPERIMENTAL METHODS AND DISCUSSION}

In our experiments we have considered the SHG from an ordered colloidal suspension of spherical polystyrene particles. In each spherical particle we encounter a lack of inversion symmetry across any given portion of the sphere surface. To enhance the second-order nonlinear interaction present at this boundary we adsorbed a layer of strongly nonlinear molecules on the surface of each sphere. Stable aqueous suspensions of negatively charged microspheres at an $8 \%$ concentration from Interfacial Dynamics Corp. were coated by dialysis with a positive chromophore of a dye molecule with a high nonlinear coefficient. The hydrophobic character of polystyrene particles, as well as the negative surface charge of each sphere, helps via the attractive Coulombian interaction the formation of a layer of nonlinear molecules with a preferred orientation of the permanent dipole moment. In our experiments we coated 0.115- and $0.137-\mu \mathrm{m}$-diam spheres with the chromophore part of Malachite green. Details of the molecular dye coated crystal preparation may be found elsewhere [10] In order to tune the Bragg refection produced by the (111) planes of the fcc lattice, the colloidal suspensions were concentrated in the dialysis membrane and then diluted appropriately.

The prepared sample was excited using an active-passive mode-locked laser emitting 35-ps pulses in the near infrared, at a wavelength of $1064 \mathrm{~nm}$. The laser pulses of an average energy of less than $5 \mathrm{~mJ}$ were focused down to a spot size 2 $\mathrm{mm}$ in diameter at the surface of the sample. The polarization of the incident beam was set to be either parallel or perpendicular to the plane of incidence and the polarization of the generated SH beam was selected using a Glann laser polarizer. The SH intensity was detected using a R212 Hamamatsu photomultiplier tube after passage through a heat absorbing filter and an interference filter centered at 532 $\mathrm{nm}$. Measurements of the reflected SH intensity when both the exciting field and the generated field at the double frequency were $p$ polarized (polarization direction parallel to the plane of incidence) are shown in Fig. 1 as a function of the angle of incidence for a crystal made of $0.115-\mu \mathrm{m}$ spheres. Such crystal shows a narrow peak with a maximum SHG intensity at $14.5^{\circ}$. Keeping the incident fundamental field $p$ polarized, the maximum $s$-polarized $\mathrm{SH}$ field was measured to be less than 500 times the intensity for the $p$-polarized SH field. Additional measurements performed after changing the fundamental field to $s$ polarization resulted in a $p$-polarized $\mathrm{SH}$ field with a 25 times smaller intensity when compared to the maximum of the $\mathrm{SH}$ peak shown in Fig. 1. However, the intensity of such a field was on average one order of magnitude larger than the $s$-polarized field generated in either configuration for the incident fundamental beam. Although similar results were obtained in transmission, a reflection geometry is considered to simplify the analysis. In such a geometry, interference with the SH light generated in the bulk of the cell walls is strongly reduced.

Efficient generation of SH light for a given transmission angle, as in Fig. 1, requires not only a contribution from each

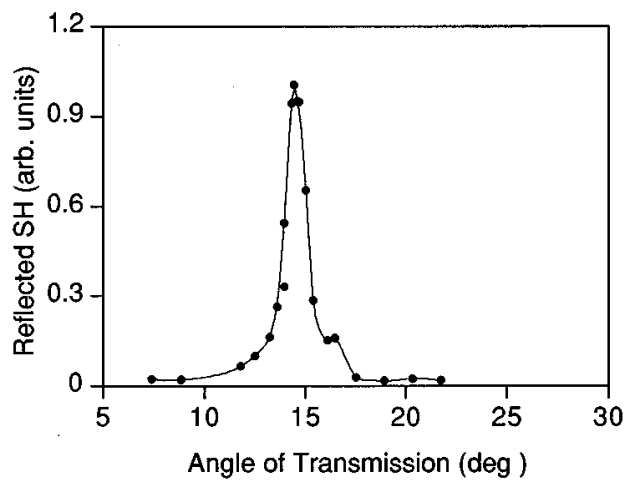

FIG. 1. Intensity of the second-harmonic light generated inside a single colloidal crystal, measured in a reflection geometry as a function of the angle of the transmitted fundamental beam relative to the normal of the (111) planes. The wavelength of the incident laser pulse was $1064 \mathrm{~nm}$. A single crystal was formed in a 1-mm-pathlength cuvette of 0.115 - $\mu$ m-diam polystyrene spheres coated with Malachite green in an aqueous suspension. The line connecting the experimental data points is a guide for the eye.

sphere in the lattice, but also phase matching of the fundamental and SH beams. In a periodic material phase matching is naturally provided by the modulation of the nonlinear susceptibility [13] or in the crystalline lattice we consider by the bending of the photon dispersion curve at the boundary of the forbidden zone [14]. Numerical calculations in a onedimensional periodic medium predict a maximum SHG in reflection or transmission for modes outside but near the forbidden zone [15]. To confirm that the bending of the photon dispersion curve near the Bragg reflection band is also the origin of the phase matching in the colloidal crystal used, we performed experimental measurements of its passive properties.

Light at $532 \mathrm{~nm}$, obtained by doubling the frequency of the laser pulse in a potassium dihydrogen phosphate $\left(\mathrm{KD}^{*} \mathrm{P}\right)$ crystal, was used to measure the reflectivity of the colloidal crystal at the frequency of the SH wave. The reflection from the (111) planes of the fcc colloidal crystal as a function of angle of incidence at a wavelength of $532 \mathrm{~nm}$ is shown in Fig. 2. Maximum reflection is obtained within a band centered where the Bragg condition $2 d \cos \theta=\lambda / n$ is satisfied. For the crystal whose SHG curve is shown in Fig. 1, the Bragg condition is satisfied at $16.7^{\circ}$, at the center of the reflection band shown in Fig. 2. Notice by comparing Figs. 1 and 2 that maximum $\mathrm{SH}$ is generated at the smaller angle side of the crystal Bragg reflection band. This is consistent with a phase matching of the fundamental and SH beams due to a decrease of roughly 0.02 in the effective index of refraction at the smaller angle edge of the Bragg reflection band. This change in the effective index of refraction for a wave propagating at the SH frequency is sufficient to overcome the phase lag between the fundamental and $\mathrm{SH}$ waves introduced by the normal dispersion of water [6].

For perfect phase matching one should expect a quadratic dependence of SHG with the length of the crystal. However, scattering losses introduced by defects in the crystalline lattice and dispersion in the spheres size severely limit the conversion efficiency of the nonlinear process. For instance, such scattering losses are seen to become larger when the sphere size is increased, reducing the efficiency of SHG as 


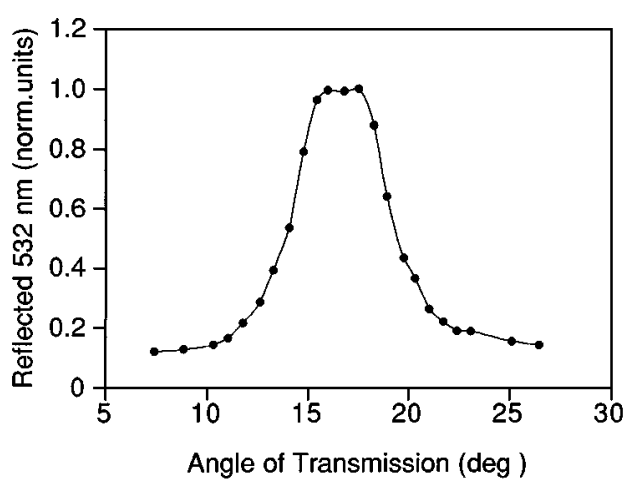

FIG. 2. Reflection of incident light at $532 \mathrm{~nm}$ as a function of the angle of the transmitted beam relative to the normal of the (111) planes for the same crystals considered in Fig. 1. The reflected intensity is normalized in the figure to the maximum reflected intensity at the angle satisfying the Bragg condition, which was measured to be on average $93 \%$. The line connecting the experimental data points is a guide for the eye.

shown in Fig. 3 for a crystal made of $0.137-\mu \mathrm{m}$ spheres (the vertical scales for Figs. 1 and 3 are given in the same arbitrary units). The Bragg reflection band corresponding to this crystal is shown in Fig. 4. Notice that although the center for the Bragg reflection band is very close to that for the crystal made of $0.115-\mu \mathrm{m}$ spheres shown in Fig. 2, the peak of maximum SHG is considerably diminished. With this reduction in the maximum intensity is a corresponding broadening of the SHG curve. Such a decrease in the length for the phase-matching range allows for the observation in Fig. 3 of a relative minimum of SHG at the large-angle side of the Bragg reflection band. At that side of the Bragg reflection band the bending of the photon dispersion curve increases the effective index of refraction, causing an extra phase mismatch, resulting in a minimum for SHG. In such imperfect crystals, actual power conversion should be approximately proportional to $L^{2} \exp (-\alpha L)$, where $L$ is the length of the crystal and $\alpha$ the extinction coefficient. Such an extinction coefficient was experimentally measured for the crystal of Fig. 2 to be $28 \mathrm{~cm}^{-1}$ at normal incidence. Using this value for the extinction coefficient in the expression above, a conversion efficiency ratio of 8 is predicted when comparing a 1 -mm-thick and a $100-\mu \mathrm{m}$-thick crystal, in close agreement

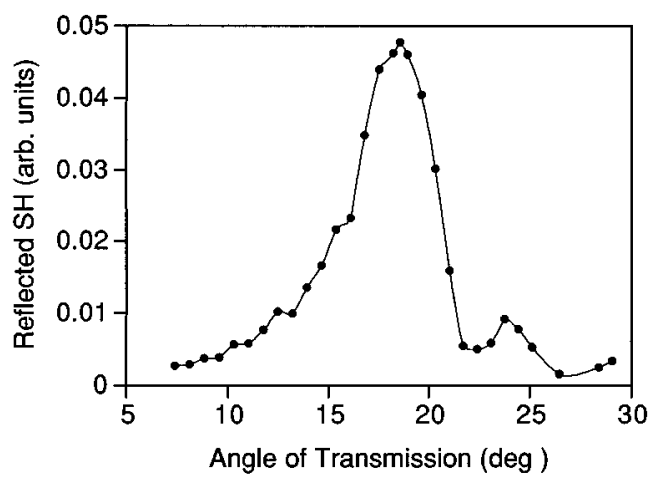

FIG. 3. Reflected SH intensity as described in Fig. 1, for a crystal fabricated with $0.137-\mu \mathrm{m}$-diam spheres. The units of the vertical scale are the same arbitrary units of Fig. 1.

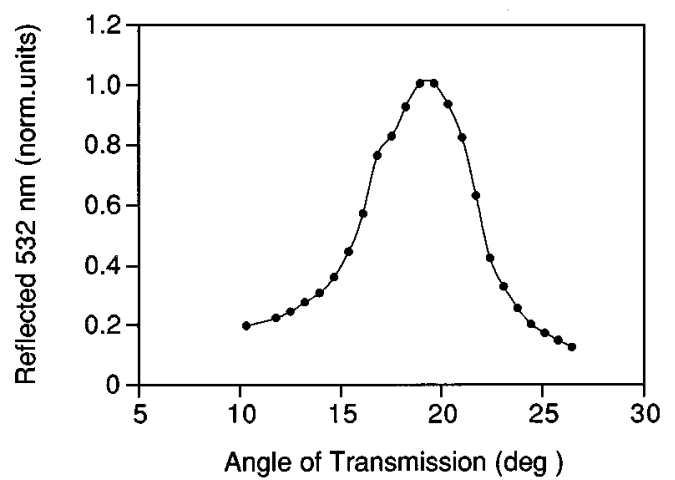

FIG. 4. Reflected light at $532 \mathrm{~nm}$ as described in Fig. 2, for the crystal fabricated with $0.137-\mu \mathrm{m}$-diam spheres considered in Fig. 3.

with the experimentally measured power conversion at the peak of maximum SHG shown in Fig. 1, which we have found to be six times larger than in a 100- $\mu$ m-thick crystal.

This behavior, in addition to the angular distribution of the reflected SH light shown in Figs. 1 and 3, clearly confirms a SHG process occurring at the bulk of the colloidal crystal. In conclusion, we attribute the experimental observation of SHG in the bulk of a centrosymmetric colloidal crystal to the nonlinear molecules adsorbed on each sphere, with the permanent dipole moment of the molecules oriented on average in the radial direction defined from the center of each sphere.

\section{THEORETICAL ANALYSIS}

To explain the observed second-order nonlinear process we first determined the spatial distribution of the SH light scattered from the layer of nonlinear material covering one dielectric sphere, which was performed by solving Maxwell's equations with a nonlinear source term. Next we considered the total field generated from a layer of spheres. An analytical solution of Maxwell's equations may be found by integration using Green's functional method of the following wave equation for the vector potential at the double frequency:

$$
\nabla^{2} \mathbf{A}+k^{2}(2 \omega) \mathbf{A}=-\mu_{0} \mathbf{J}_{\mathrm{eq}},
$$

where $k(2 \omega)$ is the wave number of the wave at the $\mathrm{SH}$ frequency and the term $\mathbf{J}_{\mathrm{eq}}$ may be considered as an equivalent current source that generates the scattered secondharmonic wave. Inside the volume of the scatterer the equivalent current source is given by

$$
\mathbf{J}_{\mathrm{eq}}=-i 2 \omega\left(\varepsilon_{0} \chi^{(1)} \mathbf{E}+\mathbf{P}^{\mathrm{NL}}\right)
$$

and is zero otherwise. The first term in this expression accounts for the linear polarizability, where $\chi^{(1)}$ is the firstorder susceptibility and $\varepsilon_{0}$ the dielectric constant of the surrounding medium, while the second part accounts for the nonlinear polarizability of the scattering medium.

In the far-field approximation and when there is no field incident at the double frequency, integration of Eq. (1) leads to the following expression for the complex amplitude of the electric field at the second-harmonic frequency, at a distance $R$ from the center of the sphere: 


$$
\begin{aligned}
\mathbf{E}(2 \omega)= & \frac{k^{2}(2 \omega) e^{i k(2 \omega) R}}{4 \pi \varepsilon_{0} R} \int\left\{\hat{\mathbf{o}} \wedge \hat{\mathbf{o}} \wedge \mathbf{P}^{\mathrm{NL}}\left(\mathbf{r}^{\prime}\right)\right\} \\
& \times e^{i[2 k(\omega) \hat{i}-k(2 \omega) \hat{\mathbf{o}}] \mathbf{r}^{\prime}} d V^{\prime}
\end{aligned}
$$

where $k(\omega)$ is the wave number of the incident fundamental wave that propagates in the direction of the unit vector $\hat{\mathbf{i}}$ and $\hat{\mathbf{o}}$ is a unit vector in the direction of the scattered $\mathrm{SH}$ wave.

The volume integral of Eq. (2) may be performed analytically by taking the field inside the scatterer at the frequency $\omega$ to be equal to the incident field in the surrounding medium or, in other words, considering the scattering in the RayleighGans regime [17]. The Rayleigh-Gans scattering approximation is valid for a scatterer whose dielectric constant relative to the dielectric constant of the surrounding medium is close to unity [17]. Under the assumptions that the dielectric sphere of diameter $D$ is covered with a homogeneous layer of nonlinear material of thickness $\delta$, the nonlinear polarizability of each nonlinear molecule in that layer is dominated by the radial component, and the frequency of the incident beam lies away from any one- or two-photon resonances of the nonlinear material, analytical integration of Eq. (2) over the homogeneous layer of nonlinear material leads to the following expression for the electric field at $2 \omega$, when the phase matching condition $k(2 \omega)=2 k(\omega)$ is satisfied:

$$
\begin{aligned}
\mathbf{E}(2 \omega)= & \frac{i k^{2}(2 \omega) e^{i k(2 \omega) R}}{R} \frac{D^{\prime}}{k_{s}} \chi_{\text {surf }}^{(2)} E(\omega)^{2}(-\sin 2 \phi \cos (\theta / 2) \\
& \times F_{1}(\theta) \hat{\mathbf{y}}_{s}+\left\{\cos ^{2} \phi \cos ^{3}(\theta / 2)\left[-F_{1}(\theta)+2 F_{2}(\theta)\right]\right. \\
& \left.\left.+\cos 2 \phi \cos (\theta / 2) F_{1}(\theta)\right\} \hat{\mathbf{o}}_{\perp}\right),
\end{aligned}
$$

where $D^{\prime}=D / 2$ is the radius of the sphere, $k_{s}$ $=2 k(2 \omega) \sin (\theta / 2)$, and $\chi_{\text {surf }}^{(2)}=\delta \rho \alpha_{r^{\prime} r^{\prime} r^{\prime}}^{(2)}$ is the surface nonlinear susceptibility, where $\rho$ is the density of nonlinear molecules and $\alpha_{r^{\prime} r^{\prime} r^{\prime}}^{(2)}$ is the only nonvanishing element of the polarizability tensor. The vector $\hat{\mathbf{y}}_{S}$ is a unit vector in the direction of the cross product of the unit vectors $\hat{\mathbf{i}}$ and $\hat{\mathbf{o}}$ and the vector $\hat{\mathbf{o}}_{\perp}$ is a unit vector perpendicular to $\hat{\mathbf{o}}$ and $\hat{\mathbf{y}}_{s}$. The angle between the incident wave vector and the scattered wave vector is $\theta$ and $\phi$ is the angle between the incident electric-field vector and the plane of scattering, a plane perpendicular to the unit vector $\hat{\mathbf{y}}_{s}$ and passing through the scattering center. $E(\omega)$ is the amplitude of the incident wave at the fundamental frequency and $F_{1}(\theta)$ and $F_{2}(\theta)$ are two scattering form factors defined as

$$
\begin{gathered}
F_{1}(\theta)=\frac{3}{k_{s}^{3} D^{\prime 3}}\left[\left(1-\frac{k_{s}^{2} D^{\prime 2}}{3}\right) \sin k_{s} D^{\prime}-k_{s} D^{\prime} \cos k_{s} D^{\prime}\right], \\
F_{2}(\theta)=\frac{3}{k_{s}^{3} D^{\prime 3}}\left[\left(1-\frac{k_{s}^{2} D^{\prime 2}}{2}\right) \sin k_{s} D^{\prime}\right. \\
\left.-\left(k_{s} D^{\prime}-\frac{k_{s}^{3} D^{\prime 3}}{6}\right) \cos k_{s} D^{\prime}\right] .
\end{gathered}
$$

By inspection of Eq. (3) and the expression of the form factors given above we see that the scattering from a single sphere at the $\mathrm{SH}$ frequency vanishes in the forward and back-

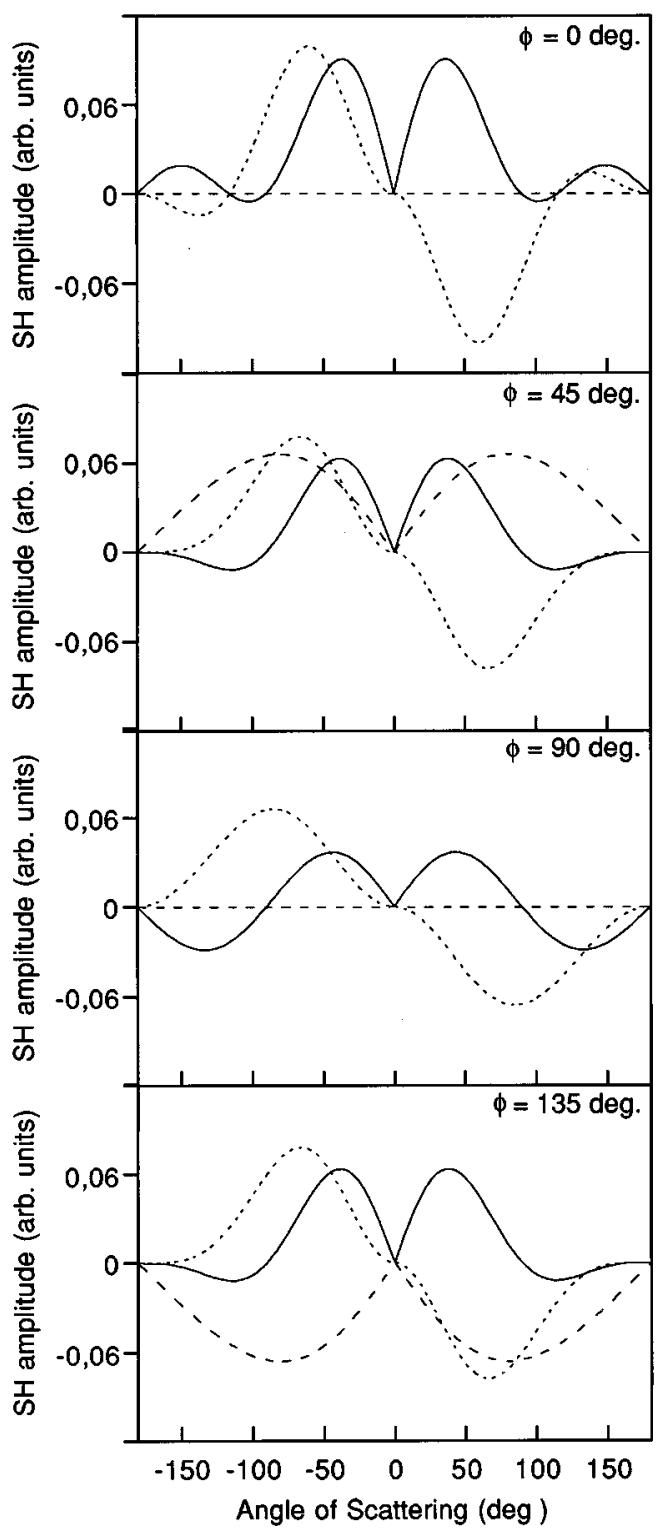

FIG. 5. Spatial distribution of the amplitude of the scattered SH light of a single sphere, for four different values of the angle $\phi$, between the polarization vector of the incident field and the plane of scattering $x-z$ as a function of the angle of scattering $\theta$. The continuous line corresponds to the $x$ component of the amplitude, the dashed line to the $y$ component, and the dotted line to the $z$ component.

ward directions (defined with respect to the propagation direction of the fundamental beam) and in general is nonzero in other directions when the light scattered at the double frequency from different portions of the sphere surface interferes constructively. This fact indicates a quadrupole character for the spatially nonuniform susceptibility responsible for the mechanism of SHG presented. Shown in Fig. 5 is the SH scattered amplitude along the axes for four values of the angle $\phi\left(0^{\circ}, 45^{\circ}, 90^{\circ}\right.$, and $\left.135^{\circ}\right)$ when the incident wave propagates along the $z$ axis, the $x-z$ plane corresponds to the scattering plane, and the spherical particle is located at the origin of the coordinate system. In this configuration $\phi$ is also the angle between the polarization vector of the incident field and the $x$ axis. Notice that the $x$ component of the $\mathrm{SH}$ 


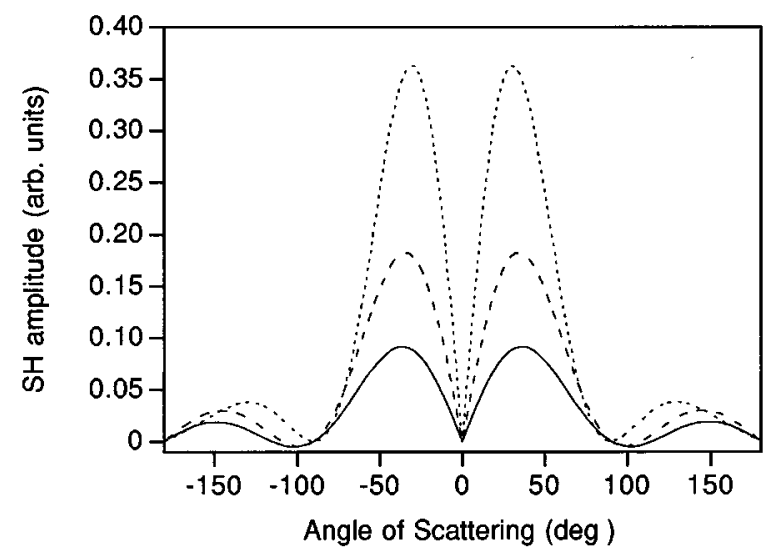

FIG. 6. Spatial distribution of the amplitude of the scattered SH light with polarization parallel to the plane of scattering of a single sphere, for three different sphere diameters, as a function of the angle of scattering $\theta$. The continuous line corresponds to a sphere diameter of $0.137 \mu \mathrm{m}$, the dashed line to a diameter of $0.2 \mu \mathrm{m}$, and the dotted line to a diameter of $0.3 \mu \mathrm{m}$.

scattered field has two lobes of the same sign, symmetric with respect to the $z$ axis. When one considers the contribution from spheres located on the $x$ axis but away from the origin, the total contribution from this type of lobe results in a nonvanishing field oriented normal to the direction of the incident beam. On the contrary, for these same spheres, the amplitude of the $z$ component of the SH field would have two lobes of opposite sign, resulting in a zero field oriented parallel to the direction of propagation of the incident wave. Contributions from all spheres located on a plane normal to the direction of the incident beam result in a plane wave [18] with a non-zero-field polarized parallel to the direction of polarization of the incident field and propagating in the direction of this incident beam. This SH field, partially reflected by the periodic distribution of dielectric material, may be detected in either a reflection or transmission geometry [15]. This prediction of the generation of a $\mathrm{SH}$ plane wave is in agreement with the experimental observation of a linear $p$-polarized SH field intensity that is 500 times larger than the measured intensity for the $s$-polarized $\mathrm{SH}$ field and propagating in the direction of the incident $p$-polarized fundamental wave. The surface character for the observed SHG process is further confirmed by the experimental observation of a SH intensity more than one order of magnitude larger for the $p$ polarization than for the $s$ polarization when the incident fundamental beam is $s$ polarized [19].

The SH amplitude parallel to the plane of scattering is shown in Fig. 6 for several values of the diameter of a spherical particle. Notice that as the diameter of the sphere becomes smaller, the amplitude of the scattered wave also diminishes, approaching zero in the limit of a particle with a small diameter when compared to the wavelength or, in other words, in the Rayleigh scattering limit, where the interference from different portions of the sphere surface is always destructive. Unfortunately, a quantitative comparison of such behavior with the experimental results is not possible since experimentally an increase in the diameter of the sphere is followed by an increase in the scattering losses, as pointed out above. Such an increase results in a transmission that for a $1-\mathrm{mm}$ crystal of $0.137-\mu \mathrm{m}$ is more than 50 times smaller than that in a crystal made of $0.115-\mu \mathrm{m}$ spheres. Such scattering losses prevent long-range phase matching in a crystal made of large spheres as indicated by the broadband for the SHG shown in Fig. 3. In conclusion, the small increase one would expect for SH scattering when using $0.137-\mu \mathrm{m}$-diam instead of $0.115-\mu \mathrm{m}$-diam spheres is completely overshadowed by the scattering losses.

The theoretical analysis presented above allows for the determination of the scattering radiation pattern for a single sphere and provides a basis to consider cooperative scattering from spheres located on a given plane, only at a qualitative level. A more quantitative analysis would require the use of Mie scattering theory. However, an alternative route to avoid the use of Mie theory would be to approximate each crystalline plane by a layer of dielectric material coated with a nonlinear molecule on both sides [20]. This last model would still hold the essential features of the SHG process observed.

\section{CONCLUDING REMARKS}

In conclusion, we have observed and theoretically interpreted a mechanism for SHG attributed to the scattering of SH light from the surface of spherical particles of optical dimensions when these particles are ordered in a crystalline lattice. We have also demonstrated that phase matching due to a change in the effective index of refractions near the edge of the Bragg reflection band of periodic structures is possible in a three-dimensional crystalline lattice of latex microspheres suspended in water. Although in the present experiment the 2.3\% dispersion in sphere size, in addition to defect such as vacancies in the crystal lattice, severely limits the conversion efficiency, in calculations using the experimental parameters and a typical value for the hyperpolarizability of a good nonlinear molecule of $10^{-27}$ esu, the theoretical model predicts a nonlinear susceptibility comparable in magnitude to the susceptibility of the commonly used KDP crystals. This value could be further increased by the deposition of several layers of nonlinear molecules on the surface of each sphere. In addition, in this mechanism of SHG, the hyperpolarizability of the molecule responsible for the nonlinear effect is not linked to a crystal symmetry that would make the phase-matching mechanism possible, as in commonly used birefringent crystals. This fact, permits a wide range of possibilities in the selection of the most appropriate nonlinear molecule. Applications are then not limited to the generation of efficient second-harmonic light and may be extended to any other nonlinear process that requires momentum matching. Aside from numerous applications in the development of new nonlinear devices, this flexibility in selecting the nonlinear molecule makes these types of structures particularly attractive in the study of surface chemical processes. Finally, we believe that the observation of the scattering of SH light from small spherical particles opens new possibilities for research in the basic field of propagation of electromagnetic waves is ordered and random media.

\section{ACKNOWLEDGMENTS}

We would like to thank F. Teixidor for useful discussions. This work was supported by Spanish DGICYT Project Nos. PB92-0600, PB93-0968, and PB95-0778. 
[1] P. A. Hiltner and I. M. Krieger, J. Chem. Phys. 73, 2386 (1969); R. Williams and R. S. Crandall, Phys. Rev. 48, 225 (1974); Roger J. Carlson and Sanford A. Asher, Appl. Spectrosc. 38, 297 (1984).

[2] Y. Kuga and A. Ishimaru, J. Opt. Soc. Am. A 8, 831 (1984); M. P. van Albada and A. Lagendijk, Phys. Rev. Lett. 55, 2692 (1985); P. E. Wolf and G. Maret, ibid 55, 2696 (1985).

[3] T. Kirkpatrick, Phys. Rev. B 31, 5746 (1985); Ping Sheng and Zhao-Quing Zhang, Phys. Rev. Lett. 57, 1879 (1986); S. John ibid. 87, 2486 (1987).

[4] A. Z. Genack, Phys. Rev. Lett. 58, 2043 (1987); J. M. Drake and A. Z. Genack, ibid. 63, 259 (1989).

[5] G. H. Watson, Jr., P. A. Fleury, and S. L. McCall, Phys. Rev. Lett. 58, 945 (1987); A. Z. Genak and N. Garcia, ibid. 66, 2064 (1991).

[6] E. Yablonovitch, Phys. Rev. Lett. 58, 2059 (1987); Jordi Martorell and N. M. Lawandy, ibid. 65, 1877 (1990).

[7] Jordi Martorell and N. M. Lawandy, Phys. Rev. Lett. 66, 887 (1991).

[8] N. M. Lawandy, R. M. Balachandran, A. S. L. Gomes, and E. Sauvain, Nature (London) 368, 436 (1994).

[9] N. M. Lawandy, S. A. Johnston, and Jordi Martorell, Opt. Commun. 65, 425 (1988).

[10] Jordi Martorell, R. Vilaseca, and R. Corbalán, Appl. Phys. Lett. 70, 702 (1997).

[11] Fielding Brown and Masahiro Matsuoka, Phys. Rev. 185, 985 (1969); T. F. Heniz, C. K. Chen, D. Ricard, and Y. R. Shen, Phys. Rev. Lett. 48, 478 (1982).

[12] K. Clays and A. Persoons, Phys. Rev. Lett. 66, 2980 (1991).

[13] J. A. Armstrong, N. Bloembergen, J. Ducuing, and P. S. Per- shan, Phys. Rev. 127, 1918 (1962).

[14] N. Bloembergen and A. J. Sievers, Appl. Phys. Lett. 17, 483 (1970); J. P. van der Ziel and M. Ilegems, ibid. 28, 437 (1976); Ammon Yariv and Pochi Yeh, J. Opt. Soc. Am. 67, 438 (1977).

[15] Jordi Martorell and R. Corbalán, Opt. Commun. 108, 319 (1994).

[16] The difference in index of refraction at the wavelengths of interest was extracted from data by I. Thormählen, J. Straub, and U. Grigull, J. Phys. Chem. Ref. Data 14, 933 (1985).

[17] In Rayleigh-Gans scattering, the wave in a given direction is the coherent addition of the waves scattered independently by each volume element of the scatterer. In this scattering regime, one may obtain simple analytical expressions for the scattered field in the far-field approximation. See, for example, H. C. Van de Hulst, Light Scattering by Small Particles (Dover, New York, 1981).

[18] The amplitude transmitted by a plane sheet of particles may be determined by dividing the plane in successive concentric Fresnel zones. The contributions to the total field from two adjacent zones are out of phase by $\lambda / 2$. See, for example, R. W. James, The Optical Principles of the Diffraction of $X$-Rays (Cornell University Press, Ithaca, 1967).

[19] At an isotropic surface or interface $s$-polarized SH radiation is not allowed under excitation of pure $s$ - or $p$-polarized light. See, for example, T. F. Heinz, Nonlinear Surface Electromagnetic Phenomena, edited by H.-E. Ponath and G. I. Stegeman (North-Holland, Amsterdam, 1991), Chap. 5.

[20] Jordi Martorell (unpublished). 